\title{
Tablet and web-based audiometry to screen for hearing loss in adults with cystic fibrosis
}

\author{
Anitha Vijayasingam, ${ }^{1}$ Emily Frost, ${ }^{2}$ Julie Wilkins, ${ }^{2}$ Lise Gillen, ${ }^{3}$ \\ Presanna Premachandra, ${ }^{3}$ Kate Mclaren, ${ }^{1}$ Desmond Gilmartin, ${ }^{1}$ Lorenzo Picinali, ${ }^{4}$ \\ Alberto Vidal-Diez, ${ }^{5}$ Simone Borsci, ${ }^{5,6}$ Melody Zhifang Ni, ${ }^{5}$ Wai Y Tang, ${ }^{7,8}$ \\ Deborah Morris-Rosendahl, ${ }^{7,8}$ Jonny Harcourt, ${ }^{9}$ Caroline Elston, ${ }^{10} \mathrm{~N} \mathrm{~J} \mathrm{Simmonds,}{ }^{1,8}$ \\ Anand Shah (10) 1,8
}

\begin{abstract}
- Additional material is published online only. To view please visit the journal online (http://dx.doi.org/10.1136/ thoraxjnl-2019-214177).

For numbered affiliations see end of article.
\end{abstract}

\section{Correspondence to} Dr Anand Shah, Adult Cystic Fibrosis Centre, Royal Brompton and Harefield NHS Foundation Trust, London SW3 6NP, UK; s.anand@imperial.ac.uk

Received 7 October 2019 Revised 18 April 2020

Accepted 20 April 2020 Published Online First 14 May 2020

\section{Linked}

- http://dx.doi.org/10.1136/ thoraxjnl-2020-215490

Check for updates

(C) Author(s) (or their employer(s)) 2020. No commercial re-use. See rights and permissions. Published by BMJ.

To cite: Vijayasingam A, Frost E, Wilkins J, et al. Thorax 2020;75:632-639.

\section{ABSTRACT}

Introduction Individuals with chronic lung disease (eg, cystic fibrosis (CF)) often receive antimicrobial therapy including aminoglycosides resulting in ototoxicity. Extended high-frequency audiometry has increased sensitivity for ototoxicity detection, but diagnostic audiometry in a sound-booth is costly, time-consuming and requires a trained audiologist. This cross-sectional study analysed tablet-based audiometry (Shoebox MD) performed by non-audiologists in an outpatient setting, alongside home web-based audiometry (3D Tune-In) to screen for hearing loss in adults with CF.

Methods Hearing was analysed in 126 CF adults using validated questionnaires, a web self-hearing test $(0.5$ to $4 \mathrm{kHz})$, tablet $(0.25$ to $12 \mathrm{kHz})$ and soundbooth audiometry ( 0.25 to $12 \mathrm{kHz}$ ). A threshold of $\geq 25 \mathrm{~dB}$ hearing loss at $\geq 1$ audiometric frequency was considered abnormal. Demographics and mitochondrial DNA sequencing were used to analyse risk factors, and accuracy and usability of hearing tests determined.

Results Prevalence of hearing loss within any frequency band tested was $48 \%$. Multivariate analysis showed age (OR 1.127; (95\% Cl: 1.07 to 1.18; $p$ value $<0.0001)$ per year older) and total intravenous antibiotic days over 10 years (OR 1.006; $(95 \% \mathrm{Cl}$ : 1.002 to 1.010; $p$ value $=0.004$ ) per further intravenous day) were significantly associated with increased risk of hearing loss. Tablet audiometry had good usability, was $93 \%$ sensitive, $88 \%$ specific with $94 \%$ negative predictive value to screen for hearing loss compared with web self-test audiometry and questionnaires which had poor sensitivity (17\% and 13\%, respectively). Intraclass correlation (ICC) of tablet versus sound-booth audiometry showed high correlation (ICC $>0.9$ ) at all frequencies $\geq 4 \mathrm{kHz}$.

Conclusions Adults with CF have a high prevalence of drug-related hearing loss and tablet-based audiometry can be a practical, accurate screening tool within integrated ototoxicity monitoring programmes for early detection.

\section{INTRODUCTION}

Individuals with chronic lung disease such as bronchiectasis, cystic fibrosis (CF) and chronic obstructive pulmonary disease (COPD) are prone to life-threatening infections with gram-negative bacteria such as Pseudomonas aeruginosa. ${ }^{1}$ This

\section{Key messages}

What is the key question?

- Can tablet or web-based audiometry be used to screen for hearing loss in adults with cystic fibrosis?

What is the bottom line?

- Tablet-based audiometry performed by nonaudiologists in an outpatient setting can accurately screen for hearing loss in chronic lung disease.

Why read on?

- This study proposes an integrated ototoxicity monitoring programme using tablet-based audiometry to monitor the under-recognised high prevalence of hearing loss in individuals with chronic lung disease.

often necessitates repeated aminoglycoside antimicrobial therapy, which remain primary agents of choice, with potential ototoxic (cochlea/vestibulotoxicity) side effects, resulting in permanent highfrequency sensorineural hearing loss. ${ }^{2}$ Furthermore, macrolides, used widely in chronic lung disease as immunomodulatory or antimicrobial therapy and prolonged intravenous amikacin for nontuberculous mycobacteria and multi-drug resistant tuberculosis (MDR-TB) treatment have potential for significant ototoxicity. ${ }^{3-8}$

Despite widespread ototoxic antimicrobial therapy use in chronic lung disease, implementation of national and international ototoxicity monitoring recommendations in the UK is sparce and inconsistent. ${ }^{9}$ Within CF, there are a number of studies detailing prevalence of hearing loss through pure-tone audiometry, with rates of $\sim 40 \%$ to $56 \%$ in adults. ${ }^{10}$ When controlling for age, cumulative lifetime intravenous aminoglycoside dosing, had a significant negative effect on hearing. ${ }^{10}$ Several variants in the mitochondrial $12 \mathrm{~S}$ ribosomal RNA gene are additionally associated with aminoglycosideinduced hearing loss, the most common being m.1555A $>$ G. ${ }^{11}{ }^{12}$ There is, however, little data on prevalence of these variants in adult chronic lung 
disease and effectiveness of genomic screening to identify highrisk individuals. ${ }^{13}$

Despite a high prevalence of ototoxicity in CF, developing effective ototoxicity monitoring programmes (OMPs) has been problematic. A recent study has highlighted the challenges of OMP implementation in chronic lung disease, with audiology departments often not co-located with respiratory services, hence necessitating further outpatient visits with delay and poor attendance. ${ }^{14}$ Sound-booth audiometry is costly, time and labourintensive and impractical in large cohorts on a frequent basis. This is particularly pertinent within resource-poor countries, where ototoxic effects are even more prevalent and reported in $>60 \%$ of patients receiving aminoglycoside-containing MDR-TB antimicrobial regimens. ${ }^{15}$ Furthermore, extended high-frequency audiometry, which is more sensitive in detecting early ototoxic effects, is often not routinely performed. ${ }^{16}$

There has hence been a technology drive to enable tablet, smartphone or web-based OMPs integrated within the clinical service to streamline care. Recent studies have validated this technology in healthy children and adults, but to date, there are no studies validating their use to detect hearing loss in adults with chronic lung disease. ${ }^{17-19}$ In this multicentre cross-sectional study, we analyse the accuracy of tablet and web-based audiometry performed by non-audiologists in an outpatient setting as screening tools for hearing loss in adults with CF and determine risk factors for hearing loss. We additionally sequence the MT-RNR1 gene to analyse prevalence and association of known variants with hearing loss.

\section{METHODS}

\section{Participants}

One hundred and fifty-six patients were recruited to the study from Royal Brompton and Harefield and King's College Hospitals NHS Foundation Trusts CF departments with 126 adults completing study follow-up. Exclusion criteria were pre-existing hearing impairment requiring hearing aids. Clinical demographics were obtained from electronic health records (EHRs) and the UK CF Registry. ${ }^{20}$ Total intravenous antibiotic days over the last 10 years was collected from UK CF Registry and cumulative intravenous antimicrobial dosing for aminoglycosides, available for preceding 5 years, from EHRs (see online supplementary data).

\section{Procedures}

Following recruitment, participants completed a web-based hearing test (developed within the 3D Tune-In project, (http:// 3 d-tune-in.eu/)) on any personal computer with standard earphones/headphones. ${ }^{21}$ For test usability and speed, pure-tone air conduction audiometry at only three frequencies was tested: $0.5,1,4 \mathrm{kHz}$. Test time was $\sim 10 \mathrm{~min}$.

Subsequently, during outpatient or inpatient setting, participants completed tablet-based audiometry (SHOEBOX Audiometry, Shoebox Ltd) with a non-audiologist research fellow using calibrated RadioEar DD450 circumaural headphones. Following otoscopy to ensure a clear ear canal and visible tympanic membrane, pure-tone air conduction audiometry at $0.25,0.5$, $1,2,4,6,8,10,12.5 \mathrm{kHz}$ was performed. Due to the crosssectional nature of the study alongside the median age of the adult CF cohorts with a significant number $>40$ years old where measurable hearing thresholds $>12.5 \mathrm{kHz}$ would be unlikely, extended high-frequency audiometric testing was pragmatically limited to $\leq 12.5 \mathrm{kHz}$ for tablet and formal audiometry, in whom background noise was monitored by the device and testing halted until below an accepted level. Test time was $\sim 10$ to $15 \mathrm{~min}$.

Lastly, sound-attenuated booth audiometric testing was completed at an audiology clinic (Imperial College Healthcare NHS Trust or Guy's and St Thomas' Hospitals' NHS Foundation Trust) using standard equipment: (1) Pure-tone air conduction audiometry at $0.25,0.5,1,2,4,6,8,10,12.5 \mathrm{kHz}$ (matching tablet audiometry) and (2)Pure-tone bone conduction audiometry from 0.5 to $4 \mathrm{kHz}$. Test time $\sim 15$ to $20 \mathrm{~min}$. A threshold of $>25 \mathrm{~dB}$ hearing loss at $\geq 1$ audiometric frequencies was considered outside normal hearing range as per current National Guidelines for Classification of Hearing Loss. ${ }^{22}$ Conductive hearing loss was defined as the presence of an air-bone gap exceeding $10 \mathrm{~dB}$ air-bone gap over three of the following frequencies 500, $1000,2000,3000$ or $4000 \mathrm{~Hz}$ (see online supplementary data).

Study participants additionally completed validated questionnaires to evaluate the impact of hearing loss on everyday tasks (Hearing Handicap Inventory for Adults (HHIA)), ${ }^{23}$ vestibular toxicity (Vertigo Handicap Questionnaire) ${ }^{24}$ and tinnitus (Tinnitus Handicap Questionnaire). ${ }^{25}$

\section{Patient experience and usability analysis}

To evaluate test usability, three standardised scales were applied to evaluate user experience and perceived usability: the User Experience Questionnaire (UEQ), ${ }^{26}$ the Usability Metric for User Experience (UMUX) ${ }^{27}$ and the Net Promoter Score (NPS) 2829 (see online supplementary data).

\section{Mitochondrial SNP analysis}

DNA was extracted from EDTA blood samples from 124 study participants. Sequencing of the MT-RNR1 gene was performed by PCR and Sanger sequencing of the two overlapping fragments spanning the entire gene. Results were visualised using Unipro UGENE 1.32 and sequences mapped to the reference mitochondrialDNA (mtDNA) genome (NC_012920.1). ${ }^{30}$ All sequence variants observed in patient samples within the sequenced region were recorded (see online supplementary data).

\section{Statistical analysis}

Results were presented as mean $\pm \mathrm{SD}$, median and IQR if nonparametric or percentage if appropriate. Univariate logistic models were fitted for each of the covariates of interest, including the allele frequency of each of the observed mtDNA variants. Univariate analysis was performed for each covariate of interest, and variables with $\mathrm{p}$ values $<0.2$ were used in the multivariate model. A stepwise selection method was used to obtain the best combination of variables. Multivariate models were fitted using the variable age as continuous. Time interaction of intravenous antibiotic use was modelled by creating a weighted score as the sum of $(1 / x$ years ago $) \times$ number of intravenous days $x$ years ago. Sensitivity, specificity, positive and negative predictive values were used to analyse accuracy of tablet and web-based audiometry using sound-booth audiometry as gold-standard. Intraclass correlation and Bland-Altman plots were used to determine reliability of tablet-based audiometry compared with sound-booth audiometry. Monte Carlo simulation was used to compare costefficiency of different screening strategies (see online supplementary data). For usability analysis, reliability of the scales were estimated using Cronbach's Alpha and paired t-test used to analyse differences between tablet, web and formal sound-booth audiometry. P values $<0.05$ were deemed significant.

\section{RESULTS}

Overall prevalence of hearing loss in our cohort at any frequency band tested through sound-booth audiometry was $45 \%(n=57)$. 

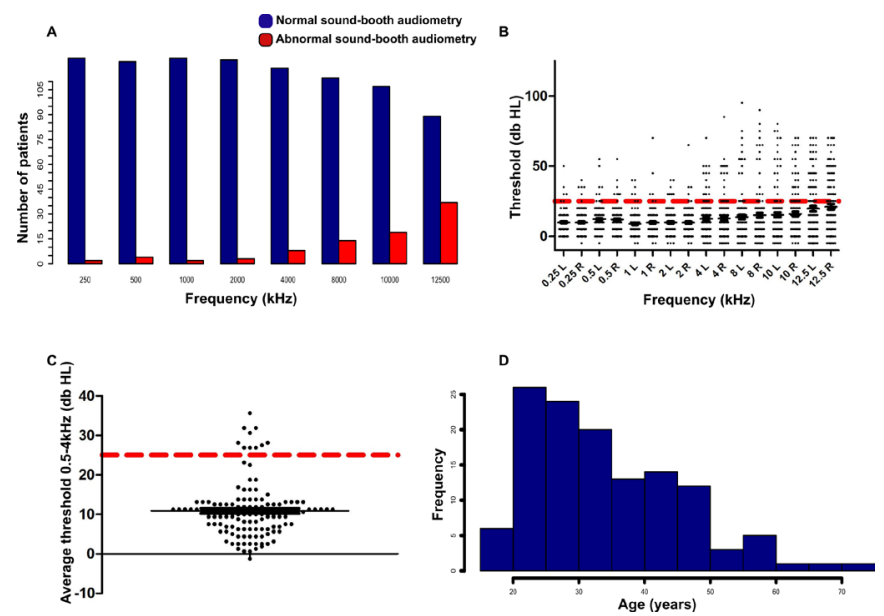

Figure 1 (A) Graph showing overall prevalence of hearing loss detected by formal sound-booth audiometry in study cohort with predominant abnormality in extended high frequencies. (B) Scatter plot showing individual thresholds for study subjects at each frequency through sound-booth audiometry to illustrate severity. Dotted red line represents cut-off for abnormality ( $\geq 25 \mathrm{~dB}$ ). (C) Graph showing average thresholds within speech discriminatory frequencies ( 0.5 to 4 $\mathrm{kHz}$ ) through sound-booth audiometry for study subjects. Dotted red line again represents cut-off for abnormality ( $\geq 25 \mathrm{~dB}$ ). (D) Bar chart representing age breakdown of study cohort and highlighting significant proportion of individuals over 40 years old.

Hearing loss was predominantly sensorineural with only $5 \%$ of abnormal hearing due a conductive defect $(n=3)$ and a further $9 \%(n=5)$ having a mixed pathology. The median age of our cohort was 31.5 (IQR 24 to 42) (figure 1). Similar to previous studies, the majority of hearing loss was at higher frequencies $(\geq 8 \mathrm{kHz})$, however, significant hearing loss was seen across all frequencies (figure 1B). A breakdown of hearing loss by frequency range is shown in online supplementary figure 1A. To determine effect on quality-of-life, thresholds were averaged across frequencies critical for speech recognition/development $(0.5$ to $4 \mathrm{kHz})$. Ten $(8 \%)$ of our cohort had abnormal average thresholds in this range (figure $1 \mathrm{C}$ ), suggesting likely significant impact on quality-of-life. Online supplementary table 1 shows demographics associated with study subjects with abnormal average thresholds critical for speech recognition/development (0.5 to $4 \mathrm{kHz})$. Online supplementary figure $1 \mathrm{~B}$ compares hearing in the 20- to 29-year-old subjects in our adult CF cohort to comparable data from a large recent study of hearing in a normal healthy population. ${ }^{31}$ This shows significantly increased average thresholds at 1, 2, 4 and $8 \mathrm{kHz}$ in our CF cohort at an age where presbycusis is unlikely.

Table 1 presents cohort clinical demographics alongside univariate association with hearing loss. Maintenance azithromycin use, age, forced expiratory volume in $1 \mathrm{~s}$ and total intravenous antibiotic days over preceding 10 years were associated with increased hearing loss. Within multivariate analysis, only age and total intravenous antibiotic days in the preceding 10 years were significantly associated with hearing loss, with covariate age explaining most of the heterogeneity in the model. For each year older the probability of an abnormal sound-booth audiometry test increases $\mathbf{1 2 . 8 \%}$ (OR 1.127 ; 95\% CI: 1.074 to $1.182 ; \mathrm{p}$ value $<0.0001)$. Despite adjustment for age, for each extra intravenous antibiotic day, the probability of an abnormal audiometry test increases $\mathbf{0 . 6 \%}$ (OR 1.006; 95\% CI: 1.002 to

Table 1 Clinical demographics of study cohort and univariate association with abnormal sound-booth audiometry

\begin{tabular}{|c|c|c|c|c|}
\hline & $\begin{array}{l}\text { All } \\
(n=126)\end{array}$ & $\begin{array}{l}\text { Normal sound-booth } \\
\text { audiometry }(n=69)\end{array}$ & $\begin{array}{l}\text { Abnormal sound-booth } \\
\text { audiometry }(n=57)\end{array}$ & $\begin{array}{l}\mathrm{P} \text { value } \\
\text { univariate } \\
\text { analysis }\end{array}$ \\
\hline Sex (n (\%) female) & $52(41.3)$ & $27(39.1)$ & $25(43.8)$ & 0.59 \\
\hline Chronic Pseudomonas aeruginosa colonisation ( $\mathrm{n}(\%))$ & $74(58.7)$ & $44(63.8)$ & $30(52.6)$ & 0.46 \\
\hline Previous history of fungal disease treatment $(\mathrm{n}(\%))$ & $49(38.9)$ & $31(44.9)$ & $18(31.9)$ & 0.13 \\
\hline Previous history of non-tuberculous mycobacterial treatment $(n(\%))^{*}$ & $25(19.8)$ & $15(21.7)$ & $10(17.5)$ & 0.56 \\
\hline Pancreatic insufficiency ( $(\%))$ & $114(90.5)$ & $64(92.8)$ & $50(87.7)$ & 0.34 \\
\hline Chronic rhinosinusitis (n (\%)) & $41(32.5)$ & $19(27.5)$ & $22(38.6)$ & 0.19 \\
\hline eGFR $(\%>90)(n(\%))$ & $90(71.4)$ & $54(78.2)$ & $36(63.2)$ & 0.05 \\
\hline Maintenance azithromycin (\%yes) & $80(63.5)$ & $38(55.1)$ & $42(73.7)$ & 0.03 \\
\hline HHIA score (n (\%) abnormal) & $14(11.1)$ & $7(10.1)$ & $7(12.3)$ & 0.70 \\
\hline Tinnitus score (n (\%) abnormal) & $12(9.5)$ & $6(8.7)$ & $6(10.5)$ & 0.73 \\
\hline Dizziness score (n (\%) abnormal) & $9(7.1)$ & $6(8.7)$ & $3(5.3)$ & 0.46 \\
\hline Age (median (IQR)) & 31.5 (24 to 42 ) & 30 (24 to 34$)$ & $42(29$ to 49$)$ & $<0.0001$ \\
\hline FEV1 (median (IQR)) & 2.1 (1.54 to 2.92$)$ & 2.56 (1.7 to 3.07$)$ & 1.85 (1.39 to 2.75$)$ & 0.008 \\
\hline FEV1 \% predicted (median (IQR)) & 62 (46 to 80$)$ & 72 (51 to 82 ) & 61 (46 to 73 ) & 0.17 \\
\hline BMI (median (IQR)) & 22 (20.1 to 23.8) & 21.5 (20 to 22.6$)$ & 22.3 (20.4 to 24.6$)$ & 0.07 \\
\hline Total days of intravenous antibiotics in the past 10 years (median (IQR)) & 56 (21 to 154$)$ & 56 (28 to 119$)$ & 56 (28 to 182$)$ & 0.03 \\
\hline †Total doses intravenous tobramycin (median (IQR)) & 28 (6 to 75$)$ & 28 (3 to 111$)$ & $28(7$ to 66.5$)$ & 0.08 \\
\hline †Total doses inhaled tobramycin (median (IQR)) & $224(0$ to 2240$)$ & 56 (0 to 1344$)$ & 938 (0 to 3001) & 0.07 \\
\hline †Intravenous amikacin (binary) \% & 17.5 & 17.9 & 16.4 & 0.47 \\
\hline
\end{tabular}

*Includes any treatment post CF diagnosis.

tRetrospective antimicrobial prescribing data only available in $n=114$ for preceding 5 years through electronic health records.

BMI, body mass index; eGFR, estimated glomerular filtration rate; FEV1, forced expiratory volume in $1 \mathrm{~s}$; HHIA, Hearing Handicap Inventory for Adults. 
1.010; p value $=0.004)$, representing a $6.7 \%$ (OR 1.067; 95\% CI: 1.028 to 1.966$)$ increased probability following a standard 14-day intravenous antibiotic course. The association, however, is predominantly driven by outliers with hearing loss and high historical intravenous antibiotic use, with similar average total intravenous antibiotic days over 10 years in those with and without hearing loss. Multivariate analysis to determine the effect of time of intravenous antibiotic use was performed by creating a weighted score as the sum of $(1 / x$ years ago $) \times$ number of intravenous days $x$ years ago. This revealed a significant time effect of intravenous antibiotic administration on ototoxicity ( $p$ value $=0.0043$; OR 1.015 (95\% CI: 1.005 to 1.026 ).

Analysis of total intravenous tobramycin doses over the preceding 5 years revealed a trend towards reduced administration in those with abnormal hearing loss. This reflects a number of subjects not receiving intravenous aminoglycosides in this time-period due to known pre-existing hearing loss. Hearing questionnaire scores were not significantly associated with hearing loss. Prevalence of significant vestibular toxicity and tinnitus symptoms were $9.5 \%$ and $7.1 \%$, respectively, which is far greater than the expected UK prevalence, but was not significantly associated with hearing loss. ${ }^{32}$ Multivariate analysis again revealed age ( $\mathrm{p}$ value $=0.038$; OR 1.063 ; 95\% CI: 1.003 to $1.227)$ and total intravenous antibiotics over 10 years $(p=0.004$; OR 1.118 ; 95\% CI: 1.037 to 1.205 ) were significantly associated with tinnitus. No variables were significantly associated with vestibular toxicity within multivariate analysis.

Given the study analysed extended high-frequency audiometry within an adult cohort where age-related hearing loss (presbycusis) is a possible aetiology, we further explored the effect of age on hearing. Figure 1D provides a graphic cohort age representation with a significant proportion (33\%) of individuals $\geq 40$ years, where presbycusis may account for hearing loss. ${ }^{31}$ The study cohort is representative of the total adult CF cohort at the Royal Brompton adult CF centre, which has a median age of 32 (range 16 to 80 ). Figure $2 \mathrm{~A}$ is a density plot showing the association between age and hearing loss with a gradual increase in hearing loss prevalence as age approaches 40 . Using a multivariate model with age as a binary variable with $\geq 40$ as a cutoff, the OR of abnormal hearing in individuals with CF $\geq 40$ compared with $<40$ years old is 28.6 (95\% CI: 8.3 to 100 ). Although age is clearly a significant factor in hearing loss development, prevalence of hearing loss in individuals $<40$ years was still high (28.6\%). Fifty per cent of individuals with abnormal average thresholds in speech discriminatory frequencies $(0.5$ to 4 $\mathrm{kHz}$ ) were again under 40 years ( $4 \%$ of total cohort). Figure $2 \mathrm{~B}$ is a density plot showing the association between total intravenous antibiotic days over the preceding 10 years and hearing loss. Although significant within multivariate modelling, there is no clear cut-off useful for screening purpose.

Forty-two different mtDNA variants in the sequenced MT-RNR1 gene region were observed in our cohort. Thirtyseven variants were single nucleotide tpolymorphisms (SNPs), whereas five were small indels (online supplementary table 2). No m.1555A $>$ G variants were detected, but the known mitochondrial variant m.827A $>$ G, previously associated with aminoglycoside ototoxicity, was found in a single individual with normal hearing, despite previous aminoglycoside exposure. ${ }^{33}$ Six novel mtDNA variants were observed, of which two variants, m.489T $>$ C (rs28625645) and m.1719G $>$ A (rs3928305) showed significant differences in allele frequency between individuals with normal and abnormal hearing. The m.489T $>C$ variant was significantly more frequent in individuals with normal hearing, while the $\mathrm{m} .1719 \mathrm{G}>\mathrm{A}$ variant was present at
A
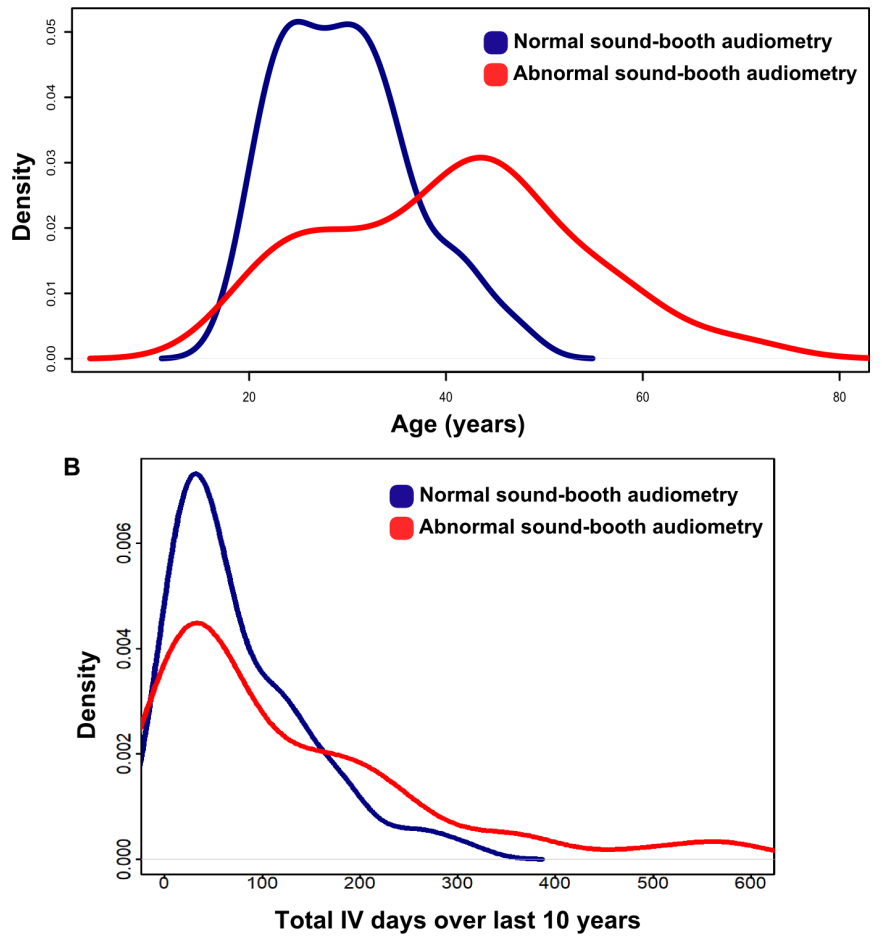

Figure 2 (A) Density plot showing association between age and abnormal hearing in study cohort. Peak prevalence in hearing loss is seen in subjects over 40 years old. (B) Density plot showing association between total intravenous antibiotic days over the preceding 10 years and hearing loss. No clear cut-off is seen which would be useful for screening stratification. IV, intravenous.

the expected population allele frequency in individuals with normal hearing $(2.99 \%)$, but was more common in those with abnormal hearing $(14.04 \%, \mathrm{p}=0.043)$.

Table 2 shows the accuracy of tablet and web-based audiometry and HHIA questionnaire to detect hearing loss using soundbooth audiometry as gold standard. Tablet audiometry showed high negative predictive value in screening for hearing loss with additional good sensitivity, specificity and positive predictive value. In contrast, although web-based audiometry had high specificity, given the limited frequencies tested, had poor sensitivity and negative predictive value. Similarly, HHIA questionnaire had high specificity but poor sensitivity and negative predictive value. Given the significant effect of age on hearing loss, we performed a Monte Carlo simulation to compare the cost-efficiency between universal screening with sound-booth or tablet audiometry or limiting tablet audiometry screening to adults $<40$ years old given the increased probability of hearing loss above that age. Universal sound-booth audiometry screening in a sample cohort $(n=539)$ would cost $£ 80$ 850. Universal tablet-audiometry screening would cost on average $£ 39605$ (95\% CI: 34550 to 45 050). Limited tablet screening to adults $<40$ years old would cost on average $£ 45611$ (95\% CI: 41300 to 50450 ). Universal tablet audiometry screening appears to be the most cost-efficient because of the true negative patients $\geq 40$ years old that could avoid sound-booth audiometry testing, in whom there would be an added socio-economic benefit with reduced outpatient visit requirement.

Intraclass correlation and Bland-Altman plots were used to further analyse tablet-based audiometry reliability compared with sound-booth audiometry. Figure 3 illustrates the high 
Table 2 Accuracy of tablet and web-based audiometry and validated symptom questionnaire (HHIA) to detect hearing loss in study cohort

\begin{tabular}{llll}
\hline & Tablet audiometry & $\begin{array}{l}\text { Web-based } \\
\text { audiometry }\end{array}$ & $\begin{array}{l}\text { HHIA } \\
\text { questionnaire }\end{array}$ \\
\hline $\begin{array}{l}\text { Apparent } \\
\text { prevalence }\end{array}$ & $0.48(0.39$ to 0.57$)$ & $0.07(0.03$ to 0.12$)$ & 0.11 (0.06 to 0.18$)$ \\
\hline $\begin{array}{l}\text { True prevalence } \\
\text { Sensitivity }\end{array}$ & $0.45(0.39$ to 0.54$)$ & $0.45(0.37$ to 0.55$)$ & $0.45(0.36$ to 0.54$)$ \\
\hline $\begin{array}{l}\text { Specificity } \\
\begin{array}{l}\text { Positive predictive } \\
\text { value }\end{array}\end{array}$ & $0.93(0.83$ to 0.98$)$ & $0.14(0.06$ to 0.26$)$ & $0.12(0.05$ to 0.24$)$ \\
\hline $\begin{array}{l}\text { Negative predictive } \\
\text { value }\end{array}$ & $0.94(0.76$ to 0.94$)$ & $1.00(0.63$ to 1.00$)$ & $0.50(0.23$ to 0.77$)$ \\
\hline
\end{tabular}

95\% Cls shown in brackets.

HHIA, Hearing Handicap Inventory for Adults.

correlation $(>0.9)$ between tablet and sound-booth audiometry at all frequencies $\geq 4 \mathrm{kHz}$ where most of the hearing loss was found. Reliability reduced at lower frequencies likely because of the effects of background noise. Representative Bland-Altman plots are shown in online supplementary figure 2 .

Figure 4 and online supplementary table 3 shows usability analysis of sound-booth, tablet and web-based audiometry. Tablet audiometry seems equivalent to sound-booth audiometry in terms of perceived usability (UMUX $=78, \mathrm{~B}+$ ) but better in terms of intention-to-use and promote $(\mathrm{NPS}=+68)$. Although, web-based audiometry appears an acceptable solution (UMUX $=72, \mathrm{C}+$ ) with a positive intention-to-promote $(\mathrm{NPS}=+11)$, it is less satisfactory than the others and is outperformed by sound-booth audiometry (UMUX $=77.5, \quad B+$; NPS $=+62$ ). The UEQ results confirm this and conversely show sound-booth and tablet-based audiometry provides a similar experience of use. Although sound-booth audiometry seems more dependable to end users, it appears less acceptable in terms of stimulation and novelty compared with tablet audiometry. Analysis also highlights a tendency of users to prefer tablet audiometry in terms of intention-to-promote compared with sound-booth testing.

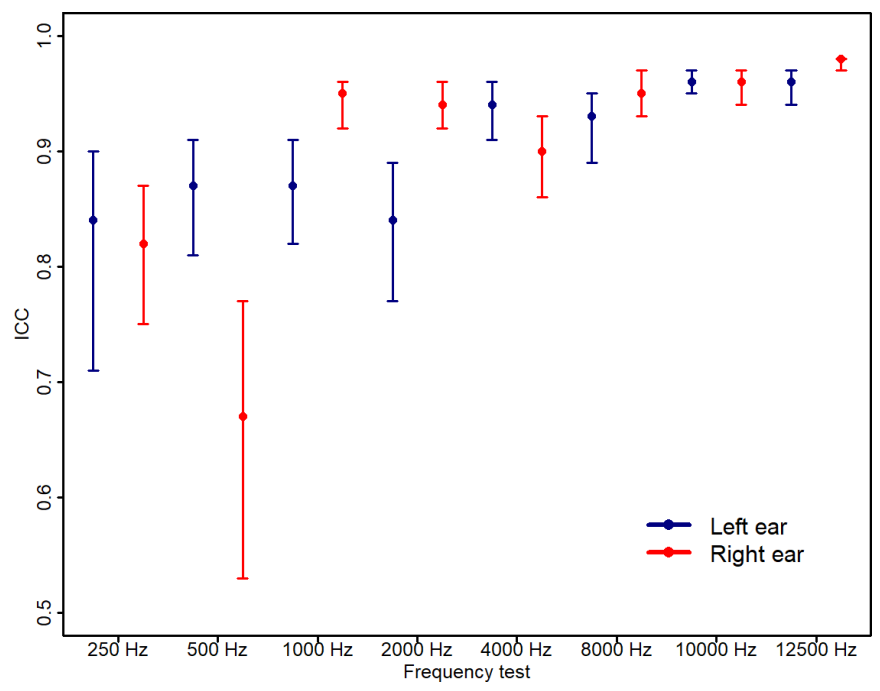

Figure 3 Intraclass correlation (ICC) analysis between tablet and sound-booth audiometry.
A

Formal Sound Booth Audiometry

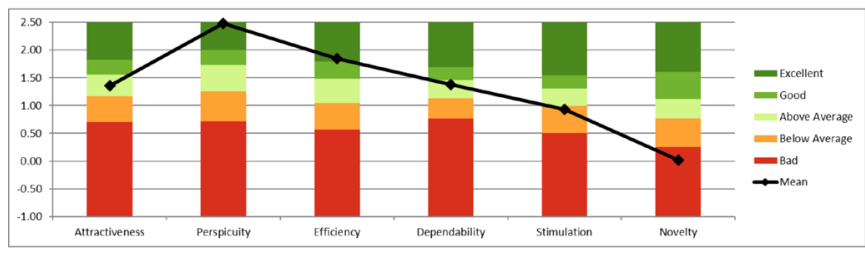

B

Tablet-based Audiometry

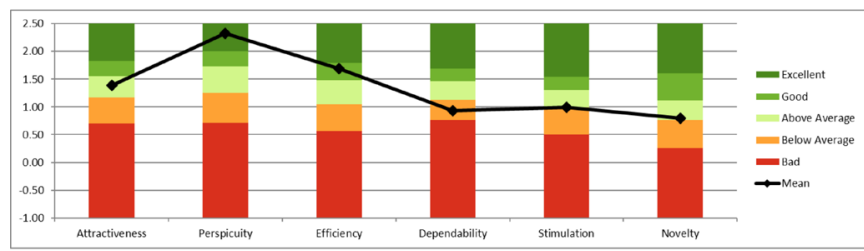

C

Web-based Audiometry

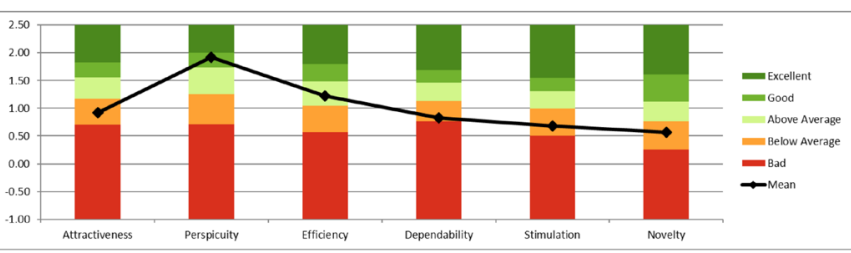

D

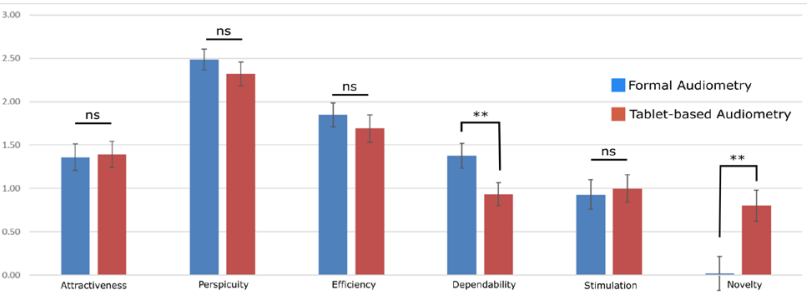

Figure 4 Graphic representation of User Experience Questionnaire (UEQ) results of (A) sound booth, (B) tablet-based and (C) webbased audiometry. Results show that web-based audiometry is less appreciated with low levels of attractiveness, dependability, stimulation and novelty. Conversely, sound booth and tablet-based audiometry seem to provide a similar experience of use. (D) Comparative analysis of the experience (UEQ) in performing tablet-based audiometry or formal sound booth audiometry. ( ${ }^{* *} \mathrm{p}<0.01$; NS, not significant).

\section{DISCUSSION}

Our study similar to those previously reported, highlights a significant prevalence of sensorineural hearing loss (48\%) in adults with CF, with a proportion of individuals ( $8 \%$ of study cohort) with severe hearing loss in frequency ranges that will affect speech discrimination and quality of life. ${ }^{10}$ Studies have highlighted the cognitive implication and effect on quality of life from severe ototoxicity-related sensorineural hearing loss in children. ${ }^{34-36}$ To the authors' knowledge, despite the high prevalence in adults with chronic lung disease, no studies evaluating the effect of ototoxicity on quality of life have been performed.

Our data reconfirms the evidence for routine hearing evaluations with extended high frequency $(>8 \mathrm{kHz})$ to detect early ototoxicity. As previously shown, age is a significant factor in determining likelihood of sensorineural hearing loss, particularly in individuals $>40$ years old. This is in line with previous studies reporting increased ototoxicity in older patients receiving aminoglycosides both with 
and without $\mathrm{CF}^{1037}$ Sensorineural hearing loss was, however, seen in a relatively large percentage of individuals $<40$ years old with a proportion again having severe hearing impairment. Age itself is a known risk factor for hearing loss through presbycusis, but within the study cohort, also represents likely cumulative antimicrobial therapy.

The total number of intravenous antibiotic days in the preceding 10 years was associated with increased risk of hearing loss, but with no clear cut-off useful for screening purpose. Lifetime cumulative aminoglycoside exposure is known to be associated with greater odds of developing sensorineural hearing loss. ${ }^{10}$ This study evaluated whether retrospective antimicrobial prescribing data easily accessible through national registry or EHRs would be useful for screening/stratification purpose, and highlights the significant limitations in available data. UK CF Registry data does not record individual aminoglycoside or antimicrobial dosing. Hence, although cumulative intravenous antibiotic days in the last 10 years was significantly associated with an increased likelihood of hearing loss in multivariate modelling, the association is relatively weak with no clear cut-off for screening purpose. Furthermore, in the centres in our study, retrospective EHR prescribing data was only available for 5 years. In an adult cohort with a median age of 31 years, this does not accurately represent cumulative life-time aminoglycoside exposure, and hence was not useful for screening stratification. In fact, given the likelihood of aminoglycoside avoidance in subjects with pre-existing confirmed hearing loss, there was a trend towards decreased aminoglycoside administration in subjects with hearing loss. Although archived information is often available, this is impractical in a clinical setting. If accurate, longerterm retrospective antimicrobial dosing is easily accessible through EHRs this may potentially enable future screening stratification through aminoglycoside exposure.

Our study also highlights the limitations in mtDNA variant genomic screening of in CF. In 124 adults, no m.1555A>G variants were identified, however, given an estimated population frequency of $0.26 \%$, this is unsurprising. ${ }^{12}$ The high overall prevalence of hearing loss, combined with a low prevalence of known mtDNA variants with increased risk of aminoglycoside-related hearing loss and reports of incomplete penetrance, suggest that mtDNA screening currently may not be cost-effective or additive to a comprehensive OMP in an adult setting. ${ }^{13} 38$ Our findings of two novel mitochondrial variants, one significantly associated with hearing loss, and the other apparently protective require confirmation in larger studies given the inability to correct for multiple testing in univariate analysis with this cohort size.

Key to preventing disabling hearing loss is an ability to perform efficient audiometric monitoring and early ototoxicity identification to enable an antimicrobial prescribing change if possible. The emergence of novel otoregenerative compounds currently in clinical trials highlight the importance for future therapeutic strategy. ${ }^{39}$ Our study shows the potential applicability of tabletbased audiometry to accurately screen for hearing loss in adults with CF with high sensitivity, specificity and negative predictive value. The approximate tariff for a formal audiogram in the UK NHS is $\sim £ 150$. As shown in our health-economic modelling, universal formal sound-booth audiometry in a cohort of $\sim 500$ individuals would cost $\sim £ 80000$ /year. In comparison, the current annual cost of universal tablet-based audiometry is modelled to be $\sim £ 40000 /$ year which represents a significant healthcare cost saving. The use of tablet audiometry in an outpatient setting by non-audiologists can enable streamlined OMP clinical integration with high uptake. The good end user usability of the device reinforces likely successful clinical care pathway integration, with a high negative predictive value enabling concentration of audiology time/expertise on high-risk individuals increasing pathway efficiency. Evaluation of real-world clinical integration including further health-economic analysis will be necessary, however, to fully determine impact. Applicability in other chronic lung disease cohorts with increased median age and resource-poor regions also requires evaluation, however, studies in older healthy individuals suggest translatability. ${ }^{40}$

Our study highlight the limitations of home web-based testing using off-the-shelf earphones, in uncontrolled (ie, potentially noisy) environments at limited frequencies (ie, speech discrimination range). Although highly specific, web-based testing had poor sensitivity in identifying hearing loss. To enable future home testing accessibility, the development of low-cost insert earphones suitable for extended high-frequency audiometry may increase reliability. ${ }^{41}$ Additionally, although useful for quality of life assessment, HHIA questionnaires were not sensitive or accurate in detecting hearing loss. The false positive incidence possibly suggests abnormal auditory processing in these subjects however further research is required to understand the use of validated questionnaires as screening tools in adult cystic fibrosis.

A limitation of our study is its cross-sectional design, given the primary outcome was to evaluate accuracy and usability of tablet/web-based audiometry compared with sound-booth audiometry. An obvious potential benefit to tablet-based audiometry, however, is an easier ability to longitudinally monitor individuals with increased ototoxicity risk. Recent studies have highlighted the ototoxic potential of a single course of aminoglycosides in $\mathrm{CF}^{42}$ This was highlighted during our study where tablet-based audiometry accurately identified early ototoxicity, enabling antimicrobial therapy change following shared decision-making (online supplementary figure 3). Further evaluation of longitudinal tablet audiometry monitoring including at increased extended high frequency up to $16 \mathrm{kHz}$ within an integrated OMP is required, but the high reliability compared with sound-booth audiometry in our study is promising. A further limitation of the study due its cross-sectional design, is the possibility of transient hearing loss detection given a small number of patients were tested while on intravenous antibiotics. Overall study hearing loss prevalence is however similar to the published literature. Lastly, given the size of the cohort we did not account for the effect of multiple testing within univariate and multivariate modelling.

Given our results, we propose an algorithm for incorporating tablet-based audiometry within an integrated OMP, using annual screening including vestibular and tinnitus questionnaires to identify individuals requiring sound-booth audiometry and evidence of ototoxicity. Tablet-based audiometry is subsequently used for longitudinal monitoring, with threshold shift modified from American Speech-Language Hearing Association guidelines used to identify ototoxic change ((i) a $15 \mathrm{~dB}$ threshold shift in (at least) one frequency OR (ii) a $10 \mathrm{~dB}$ threshold shift in two consecutive (adjacent) test frequencies), with testing frequency dependent on ongoing potential ototoxic medication use (figure 5). Further evaluation following implementation is required to determine effectiveness.

In summary, our study highlights the significant ototoxic impact of antimicrobial prescribing in adults with CF, and presents data highlighting the applicability of tablet-based audiometry as a practical screening tool with high accuracy that can be used within integrated ototoxicity monitoring programmes in chronic lung disease to identify ototoxicity at an early stage.

\section{Author affiliations}

'Adult Cystic Fibrosis Centre, Royal Brompton and Harefield NHS Foundation Trust, London, UK 


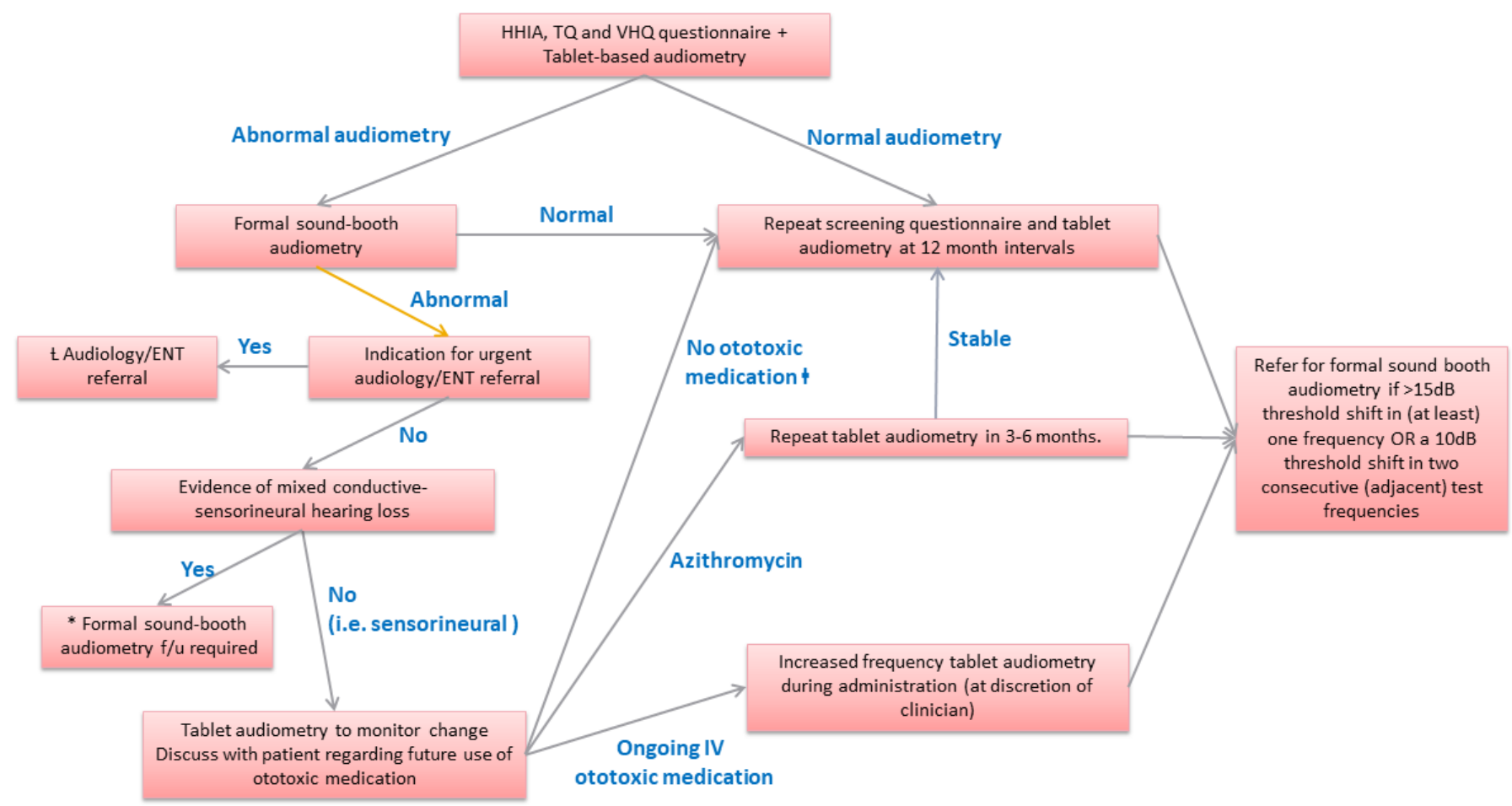

${ }^{*}$ Repeat formal audiometry at similar frequency to tablet audiometry and discuss future ototoxic medication use. Suggest referral to ENT to investigate conductive hearing loss \# Including individuals on long-standing Azithromycin with mild hearing loss

$t$ Need for referral will be indicated on formal audiometry report

Figure 5 Proposed algorithm for an ototoxicity monitoring programme incorporating tablet-based audiometry integrated within clinical care in adults with cysticfibrosis. ENT, Ear Nose and Throat; HHIA, Hearing Handicap Inventory for Adults; IV, intravenous; TQ, Tinnitus questionnaire; VHQ, Vertigo Handicap Questionnaire.

${ }^{2}$ Department of Audiology, Imperial College Healthcare NHS Trust, London, UK ${ }^{3}$ Department of Audiology, Guy's and Saint Thomas' Hospitals NHS Trust, London, UK ${ }^{4}$ Dyson School of Design Engineering, Imperial College London, London, UK ${ }^{5}$ London In Vitro Diagnostics Collaborative, Department of Surgery and Cancer, Imperial College London, London, UK

${ }^{6}$ Department of Cognitive Psychology and Ergonomics, Faculty of Behavioural Management and Social sciences, University of Twente, Enschede, Overijssel, Netherlands

${ }^{7}$ Clinical Genetics and Genomics, Royal Brompton and Harefield NHS Foundation Trust, London, UK

${ }^{8}$ National Heart and Lung Institute, Imperial College London, London, UK

${ }^{9}$ Department of Ear, Nose and Throat, Imperial College Healthcare NHS Trust, London, UK

${ }^{10}$ Department of Cystic Fibrosis, King's College Hospital NHS Foundation Trust, London, UK

Twitter Anand Shah @drAnand_Shah_

Acknowledgements The authors would like to thank the Audiology departments at Imperial College Healthcare NHS Trust and Guy's and St Thomas' NHS Foundation Trust for their support through the study. Additionally thanks to Sam Wilkinson, Lorna Briggs, Charlotte Powell and Marylka Griffiths in the Clinical Genetics and Genomics Laboratory, Royal Brompton and Harefield NHS Foundation Trust, London, UK, for technical assistance with mitochondrial DNA analysis.

Funding This study was funded through a Clinical Innovation and Excellence award from the UK Cystic Fibrosis Trust and the NHS Darzi Fellowship in Clinical Leadership programme. Alberto Vidal-Diez and Melody Ni received funding from the National Institute of Health Research (NIHR). The views expressed are those of the authors and not necessarily those of the National Health Service, the NIHR or the Department of Health.

Competing interests AS has received grant funding from Vertex Pharmaceuticals and Gilead Sciences and received personal fees for lecturing from Gilead Sciences. NJS has received personal fees for consultancy and lecturing from Vertex, Teva and Zambon Pharmaceuticals, Chiesi Ltd, Novartis International, Pulmocide Ltd, Roche and Gilead Sciences. CE has received personal fees for consultancy and lecturing from Gilead Sciences, Teva and Zambon Pharmaceuticals.

Patient consent for publication Not required.
Ethics approval Study materials and protocols were approved by the SouthCentral Research Ethics Committee (REC reference: 18/SC/0057) with informed written consent obtained at participant's clinic visit or inpatient stay.

Provenance and peer review Not commissioned; externally peer reviewed.

Data availability statement Data are available upon reasonable request. Deidentified participant data is available on request from corresponding author. ORCID ID: 0000-0001-5257-520X.

\section{ORCID iD}

Anand Shah http://orcid.org/0000-0001-5257-520X

\section{REFERENCES}

1 Faure E, Kwong K, Nguyen D. Pseudomonas aeruginosa in Chronic Lung Infections: How to Adapt Within the Host? Front Immunol 2018;9:2416.

2 Jiang M, Karasawa T, Steyger PS. Aminoglycoside-Induced Cochleotoxicity: a review. Front Cell Neurosci 2017;11:308.

3 Hansen MP, Scott AM, McCullough A, et al. Adverse events in people taking macrolide antibiotics versus placebo for any indication. Cochrane Database Syst Rev 2019;1:CD011825

4 Wang D, Fu W, Dai J. Meta-Analysis of macrolide maintenance therapy for prevention of disease exacerbations in patients with noncystic fibrosis bronchiectasis. Medicine 2019;98:e15285

5 Vermeersch K, Gabrovska M, Aumann J, et al. Azithromycin during acute chronic obstructive pulmonary disease exacerbations requiring hospitalization (BACE). A multicenter, randomized, double-blind, placebo-controlled trial. Am J Respir Crit Care Med 2019;200:857-68

6 Kwak N, Dalcolmo MP, Daley CL, et al. Mycobacterium abscessus pulmonary disease: individual patient data meta-analysis. Eur Respir J 2019:54:1-3.

7 Aznar ML, Marras TK, Elshal AS, et al. Safety and effectiveness of low-dose amikacin in nontuberculous mycobacterial pulmonary disease treated in Toronto, Canada. BMC Pharmacol Toxicol 2019;20:37.

8 Sarin R, Behera D, Khanna A, et al. Second-Line injectable induced ototoxicity in drug resistant tuberculosis: a systematic review of Indian studies. Indian J Tuberc 2019;66:279-87.

9 Maru D, Malky G-A. Current practice of ototoxicity management across the United Kingdom (UK). Int J Audiol 2018;57:S76-88. 
10 Garinis AC, Cross CP, Srikanth P, et al. The cumulative effects of intravenous antibiotic treatments on hearing in patients with cystic fibrosis. J Cyst Fibros 2017;16:401-9.

11 Jing W, Zongjie H, Denggang F, et al. Mitochondrial mutations associated with aminoglycoside ototoxicity and hearing loss susceptibility identified by meta-analysis. J Med Genet 2015;52:95-103.

12 Rahman S, Ecob R, Costello $\mathrm{H}$, et al. Hearing in $44-45$ year olds with m.1555A>G, a genetic mutation predisposing to aminoglycoside-induced deafness: a population based cohort study. BMJ Open 2012;2:e000411.

13 Veenstra DL, Harris J, Gibson RL, et al. Pharmacogenomic testing to prevent aminoglycoside-induced hearing loss in cystic fibrosis patients: potential impact on clinical, patient, and economic outcomes. Genet Med 2007;9:695-704.

14 Konrad-Martin D, Poling GL, Garinis AC, et al. Applying U.S. national guidelines for ototoxicity monitoring in adult patients: perspectives on patient populations, service gaps, barriers and solutions. Int J Audiol 2018;57:S3-18.

15 Hong H, Dowdy DW, Dooley KE, et al. Aminoglycoside-Induced hearing loss among patients being treated for drug-resistant tuberculosis in South Africa: a prediction model. Clin Infect Dis 2019;18.

16 Geyer LB, Menna Barreto SS, Weigert LL, et al. High frequency hearing thresholds and product distortion otoacoustic emissions in cystic fibrosis patients. Braz J Otorhinolaryngol 2015;81:589-97.

17 Samelli AG, Rabelo CM, Sanches SGG, et al. Tablet-Based tele-audiometry: automated hearing screening for schoolchildren. J Telemed Telecare 2020;26:18800856.

18 Rourke R, Kong DCC, Bromwich M. Tablet audiometry in Canada's North: a portable and efficient method for hearing screening. Otolaryngol Head Neck Surg 2016;155:473-8.

19 Thompson GP, Sladen DP, Borst BJH, et al. Accuracy of a tablet Audiometer for measuring behavioral hearing thresholds in a clinical population. Otolaryngol Head Neck Surg 2015;153:838-42.

20 Taylor-Robinson D, Archangelidi O, Carr SB, et al. Data resource profile: the UK cystic fibrosis registry. Int J Epidemiol 2018;47:9-10.

21 Picinali L, D'Cruz M, Simeone L, et al. 3D Tune-In: 3D-Games for tuning and learning about hearing AIDS. Intelligent Environments 2016;2016:332-7.

22 Ftouh S, Harrop-Griffiths K, Harker M, et al. Hearing loss in adults, assessment and management: summary of NICE guidance. BMJ 2018;361:361.

23 Newman $C W$, Weinstein $B E$, Jacobson GP, et al. Test-Retest reliability of the hearing handicap inventory for adults. Ear Hear 1991;12:355-7.

24 Colnaghi S, Rezzani C, Gnesi M, et al. Validation of the Italian version of the dizziness handicap inventory, the situational vertigo questionnaire, and the activity-specific balance confidence scale for peripheral and central vestibular symptoms. Front Neurol 2017;8:528.

25 Newman CW, Wharton JA, Jacobson GP. Retest stability of the tinnitus handicap questionnaire. Ann Otol Rhinol Laryngol 1995;104:718-23.
26 Laugwitz B, Held T, Schrepp M. Construction and Evaluation of a User Experience Questionnaire. Hci and Usability for Education and Work. Proceedings 2008;5298:63-76

27 Berkman MI, Karahoca D. Re-Assessing the usability metric for user experience (UMUX) scale. Journal of Usability Studies 2016;11:89-109.

28 Krol MW, de Boer D, Delnoij DM, et al. The Net Promoter Score--an asset to patient experience surveys? Health Expect 2015;18:3099-109.

29 Hamilton DF, Lane JV, Gaston P, et al. Assessing treatment outcomes using a single question: the net promoter score. Bone Joint J 2014;96-B:622-8.

30 Okonechnikov K, Golosova O, Fursov M, et al. Unipro UGENE: a unified bioinformatics toolkit. Bioinformatics 2012;28:1166-7.

31 Rodríguez Valiente A, Trinidad A, García Berrocal JR, et al. Extended high-frequency (9-20 kHz) audiometry reference thresholds in 645 healthy subjects. Int J Audiol 2014;53:531-45

32 Stohler NA, Reinau D, Jick SS, et al. A study on the epidemiology of tinnitus in the United Kingdom. Clin Epidemiol 2019;11:855-71.

33 Chaig MR, Zernotti ME, Soria NW, et al. A mutation in mitochondrial 12S rRNA, $A 827 G$, in Argentinean family with hearing loss after aminoglycoside treatment. Biochem Biophys Res Commun 2008;368:631-6.

34 Olivier TW, Bass JK, Ashford JM, et al. Cognitive implications of ototoxicity in pediatric patients with embryonal brain tumors. J Clin Oncol 2019;37:1566-75.

35 Weiss A, Sommer G, Schindera C, et al. Hearing loss and quality of life in survivors of paediatric CNS tumours and other cancers. Qual Life Res 2019;28:515-21.

36 Idstad M, Tambs K, Aarhus L, et al. Childhood sensorineural hearing loss and adult mental health up to 43 years later: results from the HUNT study. BMC Public Health 2019;19:168.

37 Gatell JM, San Miguel JG, Zamora L, et al. Comparison of the nephrotoxicity and auditory toxicity of tobramycin and amikacin. Antimicrob Agents Chemother 1983;23:897-901

38 Al-Malky G, Suri R, Sirimanna T, et al. Normal hearing in a child with the m.1555A $>$ G mutation despite repeated exposure to aminoglycosides. Has the penetrance of this pharmacogenetic interaction been overestimated? Int J Pediatr Otorhinolaryngol 2014;78:969-73.

39 Schilder AGM, Su MP, Blackshaw H, et al. Hearing protection, restoration, and regeneration: an overview of emerging therapeutics for inner ear and central hearing disorders. Otol Neurotol 2019;40:559-70.

40 Mosley CL, Langley LM, Davis A, et al. Reliability of the home hearing test: implications for public health. J Am Acad Audiol 2019;30:208-16.

41 Clark JG, Brady M, Earl BR, et al. Use of noise cancellation earphones in out-of-booth audiometric evaluations. Int J Audiol 2017;56:989-96.

42 Gleser MA, Zettner EM. Negative hearing effects of a single course of IV aminoglycoside therapy in cystic fibrosis patients. Int J Audiol 2018;57:923-30. 